\title{
POSTER
}

\section{Granulome central à cellules géantes : à propos de deux cas}

\section{Abdelqader $\mathbf{S}^{\star *}$, Roche $\mathbf{N}^{1}$, Manfredi $\mathrm{L}^{2}$, Papon $\mathrm{JF}^{2}$, Maman L' $\mathrm{L}^{1}$, Ferré $\mathbf{F}^{1}$}

1. Servie d'odontologie Charles-Foix, GH Pitié-Salpêtrière-Charles-Foix, Ivry-sur-Seine

2. Service d'ORL, GH Kremelin-Bicêtre, Villejuif, France

* Interne DESCO

\section{Introduction}

Le granulome réparateur à cellule géante ou granulome central à cellule géante (GCCG) est une tumeur bénigne intéressant quasi exclusivement les maxillaires. Elle intègre une entité nosographique de tumeurs polymorphes comprenant le kyste osseux anévrysmal, la tumeur brune de l'hyperparathyroïdie, la tumeur à cellule géante et le chérubisme. Pouvant présenter un caractère agressif, cette lésion dont la prévalence est rare et son étiologie encore inconnue atteint préférentiellement l'adulte jeune et l'adolescent. Nous rapportons ici la description et la prise en charge de deux cas de GCCR.

\section{Observations}

Le premier cas concerne une patiente de 46 ans adressée par son chirurgien-dentiste pour une tuméfaction gingivale de la mandibule persistante malgré la prise d'antibiotique. La lésion, ressentie par la patiente depuis déjà plusieurs mois présente une évolution lente et participe à une dysmétrie de l'étage inférieure. L'aspect radiologique ainsi que son caractère clinique mènent à la réalisation d'une biopsie mettant en évidence une tumeur proliférative fibroblastique contenant des cellules géantes mononuclées ainsi que des tissus ostéoïdes jeunes. Le diagnostic de GCCG est retenu et une ablation sous anesthésie locale est réalisée sans suite opératoire particulière. Dans le second cas, une patiente de 31 ans, atteinte d'une insuffisance rénale terminale, est adressée pour une tuméfaction du vestibule supérieur gauche. L'imagerie par cone-beam computed tomography (CBCT) révèle une lésion aux corticales floues et cloisonnées tandis que l'examen par IRM ne met pas en évidence d'extension aux tissus mous des fosses nasales. L'exérèse est réalisée d'emblée et son analyse anatomo-pathologique établit le diagnostic de granulome réparateur central à cellules géantes.

Discussion

Dans les deux cas les patients sont de sexe féminin et adulte. Si la première patiente ne présente pas de pathologie systémique, la seconde est atteinte d'une insuffisance rénale terminale provoquant une hyperparathyroïdie chronique. Or, l'hyperparathyroïdie peut provoquer une hyperactivité ostéoclastique à l'origine de lésions osseuses comme c'est le cas dans les tumeurs brunes de l'hyperparathyroïdisme. Bien que différents adjuvants à la chirurgie ont été proposés (injection de corticoïdes, utilisation d'interféron $\gamma, \ldots$.), le faible nombre de cas rapportés ne permet pas, pour l'heure actuelle, de proposer de traitement plus efficace que la chirurgie simple. 


\section{Conclusion}

Le caractère asymptomatique de cette lésion ainsi que son évolution lente rend sa découverte le plus souvent fortuite. Son diagnostic différentiel doit être évoqué devant une lésion ostéolytique des maxillaires dont l'étiologie n'est pas clairement établie et ce d'autant plus lorsqu'un désordre hyperparathyroïdien ou rénal complète le tableau clinique.

\section{Références}

1- De Lange Jel al. Oral Surg Oral Med Oral Path Oral Rad Endo 2005:99:464-470.

2 - Jaffe H.L. Oral Surg 1953:6:159-175.

3 - Kruse-Loster B et al. Oral Surg Oral Med Oral Path Oral Rad Endo 2006:101:346-354. 